\title{
Securin is overexpressed in breast cancer
}

\author{
Selam Ogbagabriel ${ }^{1}$, Manory Fernando ${ }^{1}$, Frederic M Waldman ${ }^{2}$, Shikha Bose ${ }^{3}$ and \\ Anthony P Heaney ${ }^{1}$ \\ ${ }^{1}$ Department of Medicine, Cedars-Sinai Research Institute, UCLA School of Medicine, Los Angeles, CA, USA; \\ ${ }^{2}$ School of Medicine, UCSF Cancer Center, San Francisco, CA, USA and ${ }^{3}$ Department of Pathology, \\ Cedars-Sinai Research Institute, UCLA School of Medicine, Los Angeles, CA, USA
}

\begin{abstract}
Securin regulates sister chromatid separation during mitosis, induces bFGF-mediated angiogenesis, and securin overexpression causes in vitro transformation and in vivo tumor formation in nude mice. As estrogen administration to oophorectomized rats increased pituitary securin expression, we used immunohistochemistry to examine securin and estrogen receptor alpha (ER- $\alpha)$ expression in 90 breast tumors and 18 normal breast tissues. Breast tumor securin and ER- $\alpha$ expression were quantitated by image analysis and expressed as fold difference relative to securin expression in normal breast tissue. Low cytoplasmic securin expression was seen in the normal breast epithelium, whereas abundant cytoplasmic and nuclear securin expression was demonstrated in all 90 breast tumors. Highest securin expression was seen in brain metastatic breast tumors (4.3-fold, $P<0.01$ ), cells derived from metastatic breast cancers $(6.5$-fold, $P<0.001$ ), and in invasive ductal carcinoma (mean \pm s.e.: 3.8 -fold, $\boldsymbol{P}<\mathbf{0 . 0 0 1}$ ). Highly pleomorphic (4.1-fold) or highly proliferative breast tumors (1.6-fold) exhibited high immunohistochemical securin expression compared to low-grade breast tumors $(P<0.05)$. Northern blot analysis in 12 of the breast tumors confirmed the immunohistochemical findings demonstrating increased securin mRNA expression compared to normal breast mucosa $(2.5$-fold, $P=0.03)$, with highest securin evident in invasive (3.5-fold) vs noninvasive tumors (1.9-fold, $P=0.03)$. In addition, some tumors that exhibited high securin expression also expressed high ER- $\alpha$ levels $(P<0.0001)$. These results demonstrate that the estrogen-induced transforming gene, securin is abundantly expressed in breast carcinoma, and is associated with the presence of metastatic spread, and lymph node invasion. We propose immunohistochemical tumor securin expression as a potential invasive marker, and novel therapeutic target in breast cancer.
\end{abstract}

Modern Pathology (2005) 18, 985-990. doi:10.1038/modpathol.3800382; Published online 22 April 2005

Keywords: breast cancer; estrogen; mitotic activity; nuclear pleomorphism; securin

Breast cancer, is the second highest cause of cancer death among women. ${ }^{1,2}$ The National Surgical adjuvant breast and bowel projects breast cancer prevention trial $(\mathrm{P}-\mathrm{I})^{3}$ and others ${ }^{4}$ have demonstrated the effectiveness of antiestrogens in reducing the risk of estrogen receptor (ER)-positive breast cancer. However, despite these advances, identifying patients with aggressive disease at onset who may benefit from adjuvant therapies remains a challenge. Although histopathological parameters such as lymph node or vascular invasion, estrogen receptor, ${ }^{5}$ progesterone receptor and Her-2/neu ${ }^{6}$ expression are helpful, additional breast tumor markers that provide prognostic insights are needed.

Correspondence: Dr AP Heaney, MD, PhD, Division of Endocrinology, Cedars-Sinai Medical Center, 8700 Beverly Blvd. B-127, Los Angeles, CA 90048, USA.

E-mail: HeaneyA@cshs.org

Received 3 November 2004; revised 17 December 2004; accepted 20 December 2004; published online 22 April 2005
Pituitary tumor-derived transforming gene (PTTG) was identified by differential display from a rat pituitary tumor. PTTG is a securin protein, which regulates chromatid separation during mitosis. ${ }^{7}$ Securin overexpression leads to aneuploidy, ${ }^{8,9}$ causes in vitro transformation, and tumor formation in vivo, regulates bFGF secretion and induces angiogenesis. ${ }^{10,11}$ Securin is expressed at low levels in normal tissues, but is abundantly expressed in human pituitary, ${ }^{12}$ thyroid ${ }^{13}$ and colorectal tumors, ${ }^{14}$ where highest securin mRNA expression was observed in invasive and metastatic cancers.

In addition, estrogen administration to oophorectomized rats led to the development of large prolactin-secreting pituitary tumors in association with elevated serum prolactin levels and a $\sim 3$-fold increase in pituitary securin mRNA expression. ${ }^{15}$ As pituitary and breast cellular proliferation are both hormone dependent, we examined securin expression in 90 breast tumors and 18 normal breast tissues. 


\section{Materials and methods}

\section{Human Subjects and Tissues}

In accordance with institutional review board human subjects research guidelines, tissue microarray (TMA) slides containing $0.6 \mathrm{~mm}$ cores from 82 individual breast tissue specimens (seven DCIS, 14 invasive lobular carcinomas, 55 invasive ductal carcinomas, six metastatic breast cancer cell lines (MDA-MB-453, MDA-MB-231, T-47D, BT-474, MCF7, SK-BR-3) were provided from the University of California, San Francisco-Specialized Program of Research Excellence (UCSF-SPORE). An additional eight brain metastatic breast cancers and 18 normal breast tissues were obtained following surgical resection and reduction mammoplasty, respectively. Tumor grade, degree of nuclear pleomorphism and mitotic activity were also available for the breast tumors. Mitotic activity was determined by counting mitosis in 10 high-power fields and graded $1-3$ as follows: up to seven mitosis, grade 1; 8-14 mitosis, grade 2 ; $\geq 15$ mitosis, grade 3 . Nuclear pleomorphism was graded using a modified Scarff-BloomeRichardson system, presence of small uniform cells, grade 1; moderate increase in nuclear size and variation, grade 2 ; marked variation in nuclear size and/or shape, grade 3 .

\section{Immunohistochemistry}

Breast tumor tissue sections $(5 \mu \mathrm{m})$ were deparaffinized, rehydrated, washed in PBS for $5 \mathrm{~min}$ and antigens retrieved in $0.01 \mathrm{M}$ sodium citrate buffer (pH 6). Sections were then incubated overnight with rabbit polyclonal anti-PTTG/Securin (Zymed, San Francisco, CA, USA) (1:200 dilution) or anti-ER- $\alpha$ (Dako Corporation, Carpinteria, CA, USA) (1:100 dilution) antibodies, washed in PBS, and incubated with a secondary biotinylated goat anti-rabbit antibody (1:200 dilution). Slides were next incubated with ABC (Vector, Burlingame, CA, USA) (1:100 dilution), washed and overlaid with DAB substrate (Biogenex, San Ramon, CA, USA) prior to color development and analysis.

Immunohistochemical securin and ER- $\alpha$ expression were quantitated using ImagePro Plus quantitative analysis software (Media Cybernetics, Silver Spring, MD, USA). Briefly, the intensity of securin and ER- $\alpha$ expression was quantified by selecting the colored field (brown) and computing the integrated optical density in arbitrary units. Breast tumor securin immunoreactivity was then expressed as fold-increase relative to the mean epithelial cell securin expression in the 18 normal breast tissues.

\section{Northern Blot Analysis}

Total RNA was extracted from a subset of 12 randomly selected excised breast tissues with
TRIzol (Invitrogen, Carlsbad, CA, USA). RNA derived from JEG-3 choriocarcinoma cells served as a positive control for securin expression. Electrophoresed RNA was transferred to Hybond-N nylon membranes (Amersham International, Buckinghamshire, UK), and hybridized at $68^{\circ} \mathrm{C}$ with human securin cDNA as previously described. ${ }^{13}$ Securin mRNA expression was quantitated by scanning densitometer (Alpha Innotech Corporation, San Leandro, CA, USA), normalized to $\beta$-actin expression and expressed as fold-increase relative to either matched normal mucosa from the same individual (eight breast cases), or the mean securin/actin ratio measured in normal breast tissues $(n=13)$.

\section{Western Blot Analysis}

Protein lysates were prepared from the same subset (used for Northern blot analysis) of breast tissues using RIPA buffer, ${ }^{15}$ soluble proteins (50 $\mu$ g) separated by SDS-PAGE electrophoresis, transferred to PVDF membranes (Amersham), and incubated with antibodies to securin (1:5000), and $\beta$-actin (1:2500; Sigma). Blots were then washed and incubated with appropriate horse radish peroxidase- conjugated secondary antibodies. Complexes were then visualized by ECL chemiluminescence detection.

\section{Statistical Analysis}

Results are expressed as the mean \pm s.e. Statistical analysis was performed using ANOVA with Bonferroni's multiple comparison $t$-test, the unpaired $t$-test or Spearman's rank correlation. $P<0.05$ was considered significant.

\section{Results}

\section{Securin is Overexpressed in Breast Tumors}

In 18 normal breast tissues, securin expression was localized predominantly in the cytoplasm of breast ductal epithelial cells (Figure 1a). In marked contrast, abundant securin expression (mean \pm s.e. foldincrease; $3.5 \pm 0.2, P<0.0001$ ) was observed in all 90 breast tumors (Figure 1b) compared to normal breast tissues and was distributed throughout the cytoplasm and in large, nuclei (Figure 1c). The heterogeneity of securin expression within the breast tumor (depicted in Figure 1) may be due to different tumor cell proliferative rates. Previous studies examining single cell mitosis have shown that securin is expressed in a cell-cycle-specific manner during which securin expression peaks at anaphase and then is degraded by ubiquitination. ${ }^{16}$ High securin expression was seen in invasive breast ductal carcinomas (mean \pm s.e. fold-increase; $3.8 \pm$ $0.3, P<0.001)$, in brain metastatic breast tumors (4.3 $\pm 0.7, P<0.01)$, with highest levels in cells derived from metastatic breast cancer $(6.5 \pm 1.3$, 
$P<0.001$ ) (Figure 1d) as compared to normal breast tissues. Although higher securin expression was seen in DCIS ( $n=7,2.3 \pm 0.4, P=\mathrm{NS})$, and invasive
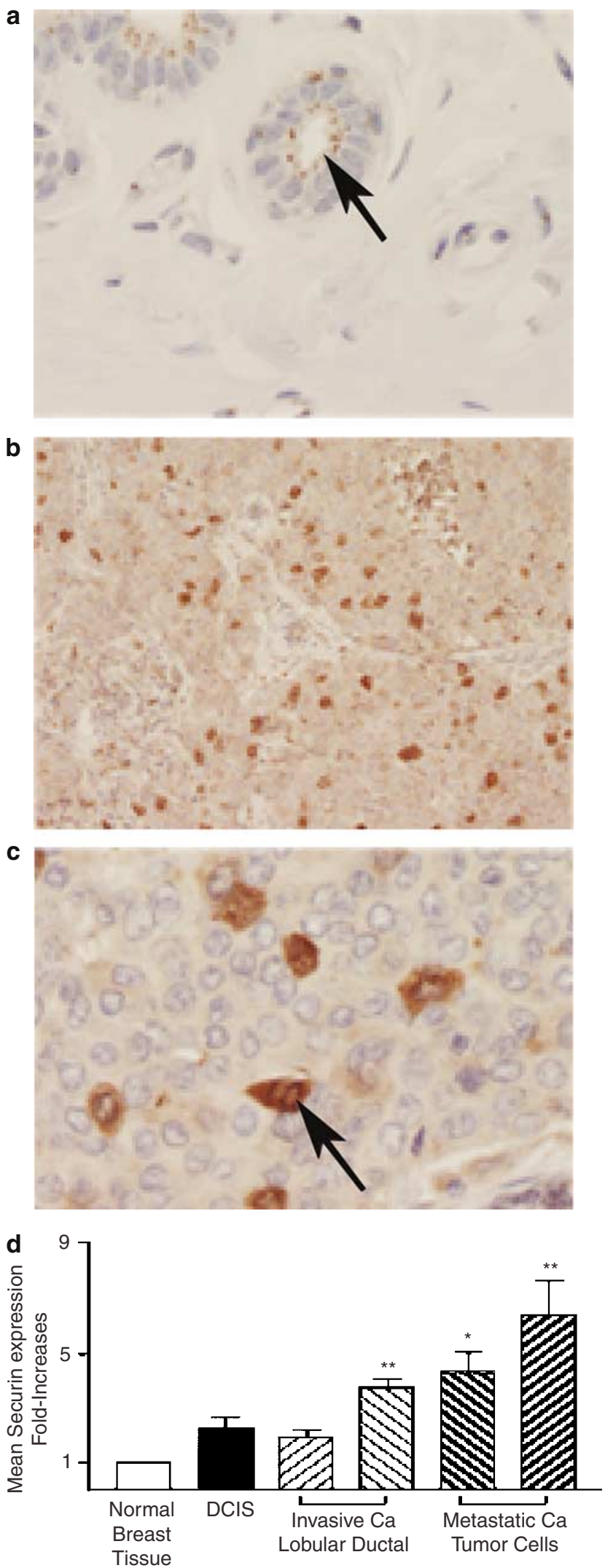

lobular breast cancer $(n=14,1.9 \pm 0.3, P=\mathrm{NS})$, in comparison to normal breast tissues, these differences did not attain statistical significance. These findings demonstrate that securin is an important marker of breast ductal cancer invasion.

In a subset of randomly selected representative breast tumors for which sufficient tissue was available, Northern blot analysis was used to compare securin mRNA expression in breast tumors (ductal carcinoma in situ, $n=2$, invasive ductal carcinoma, $n=10$ ) with securin levels in normal breast tissues $(n=6)$, derived from the same individual. In support of our immunohistochemical findings, securin mRNA expression was increased in all 12 breast carcinomas examined (mean \pm s.e. fold-increase; $2.5 \pm 0.3$ ) as compared to normal breast tissues $(0.2 \pm 0.06, \quad P<0.01)$ (Figure 2a). Furthermore, highest securin mRNA expression was observed in the three of these 12 breast tumors which exhibited evidence of lymph node invasion (mean \pm s.e. fold-increase; lymph node positive $3.5 \pm 0.45$ vs lymph node negative $1.9 \pm 0.34$, $P=0.03)$ as compared to tumors confined to the breast (Figure 2b). Western blot analysis of protein lysates derived from these same tumors supported the Northern blot findings, demonstrating high securin expression in breast tumors (Figure 2c) compared to expression in adjacent normal breast tissue. Securin overexpression correlates with mitotic index, degree of nuclear pleomorphism and ER- $\alpha$ expression.

As more invasive breast tumors exhibited the most intense securin immunostaining (Figure 1c), we next compared the intensity of breast tumor securin expression with breast tumor mitotic rates and their degree of nuclear pleomorphism. The mitotic rates and degree of nuclear pleomorphism of the breast tumors were independently determined (detailed in methods) by a pathologist (FW, UCSF-SPORE) without prior knowledge of breast tumor securin expression. Highest securin expression was seen in the breast tumors which exhibited highest mitotic activity (mean \pm s.e. fold-increase; grade $3 ; 1.6 \pm$ $0.3, P<0.05$ ) (Figure 3a) compared to grade 1 breast tumors, supporting the finding that securin is a

Figure 1 Securin is abundantly expressed in breast cancer Representative immunohistochemical securin expression in normal breast tissue demonstrated low level securin immunoreactivity in the cytoplasm of ductal epithelial cells (a) (magnification $=\times 100$ ). In contrast, abundant securin was seen in the breast cancers (b) (magnification $=\times 100$ ), (c) (magnification $=\times 400$ ) and was evident in both the cytoplasm and nucleus. Quantiative analysis of securin demonstrated higher securin levels in invasive breast cancers $(n=90)(\mathbf{d})$. Normal breast tissue $(n=18)$, DCIS $(n=7)$, invasive lobular carcinoma $(n=14)$, invasive ductal carcinoma $(n=55)$, metastatic breast cancer cell lines $(n=6)$. Immunohistochemical securin expression was quantified and expressed relative to mean epithelial securin expression in 18 normal breast tissues (details in Materials and methods) $\left({ }^{*} P<0.01 ;{ }^{*} P<0.001\right)$. 


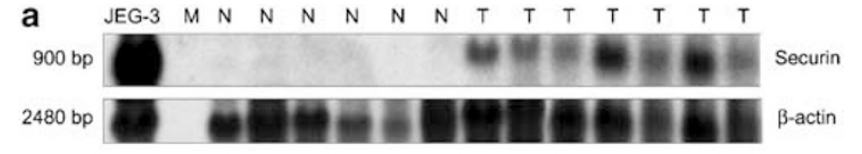

b

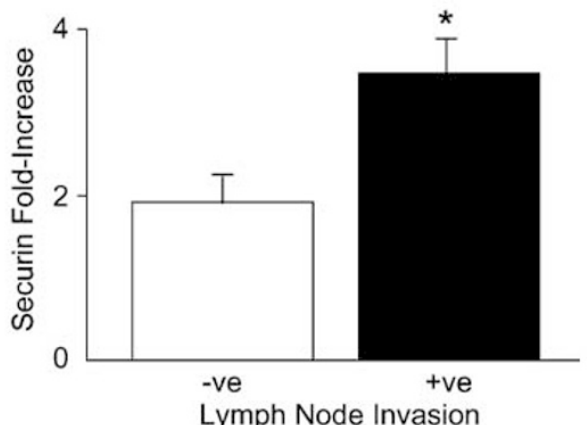

C

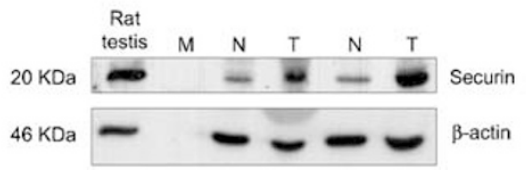

Figure 2 Representative Northern (a) blot analysis of normal (N) and tumor $(\mathrm{T})$ tissue depicting expression of securin mRNA. Quantitative securin mRNA expression (b) in the breast tumors which exhibited lymph node invasion as compared to noninvasive breast tumors $\left({ }^{*} P=0.03\right)$. Western blot analysis confirmed elevated tumor securin levels (c). Statistical analysis by ANOVA. $\beta$-actin served as RNA and protein loading controls. JEG-3 and rat testis served as positive securin controls. $\mathrm{M}=\mathrm{RNA}$ or protein size marker.

marker of breast tumor proliferation. Furthermore, highest securin expression was observed in breast tumors with the highest degree of nuclear pleomorphism (mean \pm s.e. fold-increase; grade 3 ; $4.1 \pm 1.3, P<0.05$ ) (Figure $3 \mathrm{~b}$ ) compared to grade 1 pleomorphic tumors. Given our previous studies demonstrating estrogen-mediated pituitary securing induction ${ }^{15}$ we also examined ER- $\alpha$ expression in the breast tumors. In all, 71 of 90 tumors exhibited immunohistochemical ER- $\alpha$ expression, and in most of these tumors concordant high securin and ER- $\alpha$ expression levels were observed (Figure 3c).

\section{Discussion}

An estimated 192200 women were diagnosed with invasive breast cancer in 2001, and it remains the second most common cause of cancer death in industrialized nations. ${ }^{1}$ However, despite these statistics, it has become increasingly clear that adjuvant therapy improves survival in all subgroups of women with invasive breast cancer. ${ }^{17-19}$ A considerable challenge that remains is to identify those women who will best benefit from often intensive chemotherapeutic regimens, as absolute benefit varies depending on tumor stage and other prognostic features. ${ }^{20}$
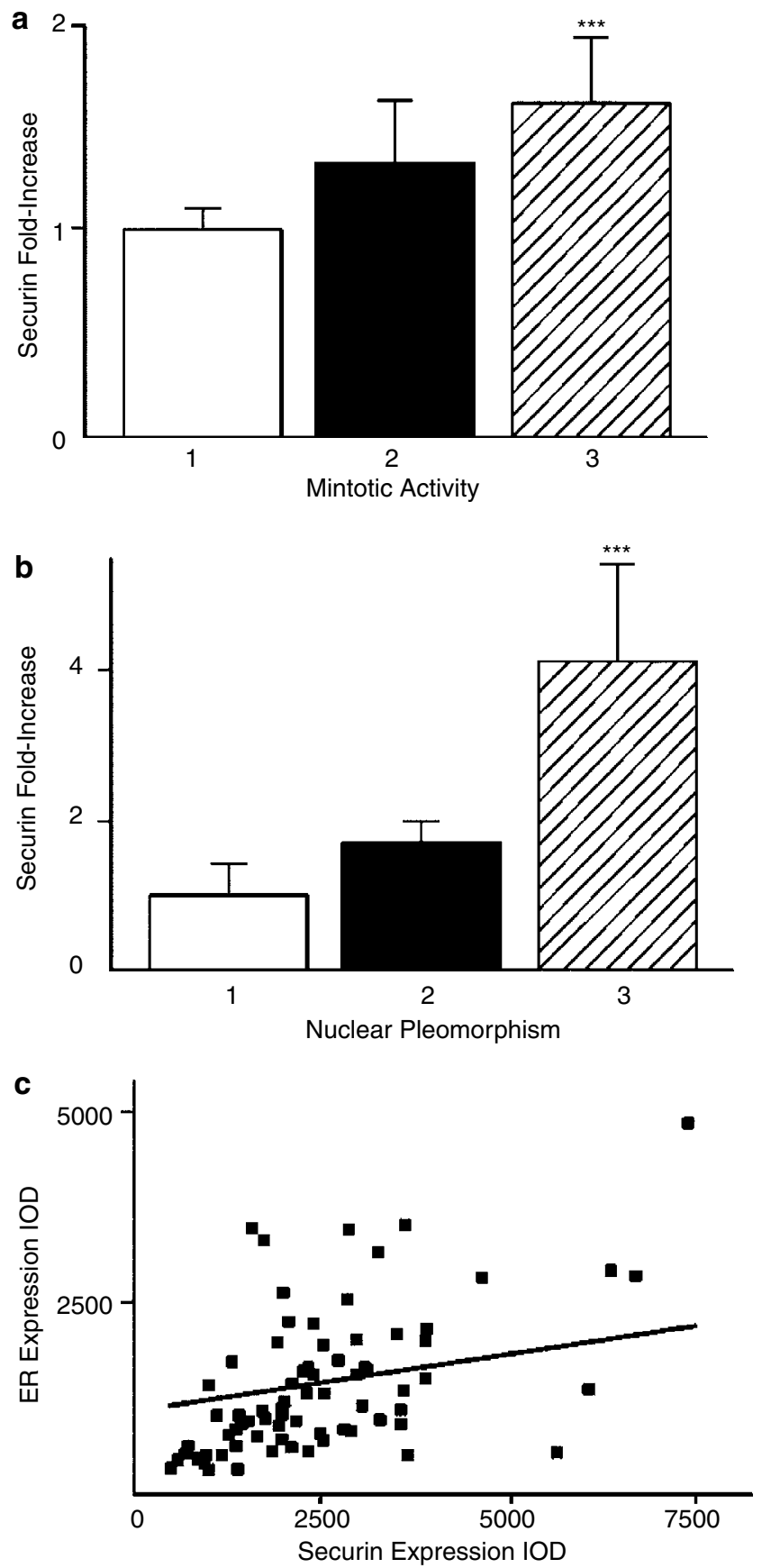

Figure 3 Graphic representation of breast tumor securin expression which correlated with mitotic index $(n=87)(\mathbf{a})$, and degree of nuclear pleomorphism $(n=87)\left(\right.$ b) $\left({ }^{* *} P<0.05\right)$. A correlation between immunohistochemical ER and securin expression was seen in some breast tumors (Spearman's $r=0.54 ; P<0.0001$ ) (c).

The premise that rapidly proliferating tumors are more dangerous than slowly dividing tumors is difficult to challenge, but the measurement of DNA content and/or S-phase fraction cannot always make this prediction. The use of proliferative markers such as Ki-67 can determine the proportion of actively dividing cells in a given tumor, but a potential disadvantage of $\mathrm{Ki}-67$ is that it is expressed 
in G1, G2 and M-phases of the cell cycle, as well as those cells in S phase. ${ }^{21,22}$

The securin protein is expressed in a cell-cycledependent manner. Following cytoplasmic to nuclear translocation, it peaks in G2/M-phase ${ }^{16}$ and thereafter is rapidly degraded by ubiquitination. ${ }^{23}$ We demonstrate here that securin is abundantly expressed in breast carcinoma compared to normal breast tissue, and highest securin expression was detected in breast tumors with the highest mitotic rates and highest degree of nuclear pleomorphism. Although we have not correlated securin expression with Ki-67 levels in these breast tumors our previous studies demonstrated that securin overexpression leads to increased proliferative rates, and mitogens such as estrogen and bFGF increased pituitary, thyroid and breast tumor securin levels. ${ }^{13,15,24}$ We propose that our observed increased securin is a marker of breast tumor proliferative capacity and may aid in detection of cancers that are more likely to follow an aggressive clinical course, and the identification of patients that are more likely to benefit from adjuvant therapies. As securin overexpression disrupts normal sister chromatid separation leading to aneuploidy, an alternate explanation for increased securin expression in the breast tumors may be due to aneuploidy.

Ductal carcinoma in situ is confined to the breast ducts, is considered premalignant, and over time may progress to invasive breast ductal carcinoma. ${ }^{25}$ Our study did not include a sufficient number of DCIS cases to perform subgroup analysis and whether securin expression in DCIS may help predict likelihood of subsequent invasion is unclear. Invasive lobular carcinoma accounts for $\sim 5-10 \%$ of breast cancers. Interestingly, comparatively lower levels of securin were demonstrated in the invasive lobular carcinomas examined. This result may in part be explained by the histology of lobular carcinoma, which is characterized by abundant fibrous tissue, that is diffusely infiltrated by uniform, small round cells, which typically exhibit lower mitotic activities than invasive ductal carcinomas, and the lower securin expression that we observed, may simply reflect lower tumor proliferative rates. ${ }^{26-28} \mathrm{~A}$ further explanation for the comparatively lower securin expression seen in lobular carcinomas compared to ductal carcinomas was that two-thirds of the lobular carcinomas exhibited a low degree of nuclear pleomorphism and only one tumor was markedly pleomorphic.

A variety of factors contribute to tumor invasion including angiogenesis, ${ }^{29}$ and we have previously demonstrated that securin overexpression in NIH 3T3 cells leads to increased bFGF secretion and angiogenesis, ${ }^{9}$ and our previous studies in colorectal cancers demonstrated that highly vascular tumors exhibited high securin expression. ${ }^{14}$ In addition to its actions on cell-cycle regulation, securinmediated angiogenesis may provide a further me- chanism whereby securin abundance contributes to an invasive breast tumor phenotype.

We have demonstrated that high ER- $\alpha$ expression correlates with high securin expression in some breast tumors but clearly some tumors with low ER expression also exhibited high securin levels, and the correlation between ER- $\alpha$ and securin expression was not observed in all tumors. Our previous studies have demonstrated that estrogen induces pituitary securin expression, but as securin is a marker of cell proliferation, other growth factors may induce breast cancer growth and coincident securin expression.

Securin overexpression in vitro results in aneuploidy that most agree contributes to the malignant phenotype. $^{30,31}$ Aneuploidy results from many diverse molecular mechanisms, including dysregulation of sister chromatid separation during mitosis. ${ }^{32}$ Recently increased MAD2, and securin levels in association with aneuploidy were observed in p53 null mammary cells following hormone stimulation, providing insight into mechanisms by which hormones such as estrogens may contribute to chromosomal mis-segregation in breast tumors. ${ }^{33}$ These studies identify these key mitotic proteins as potential novel cancer therapeutic targets. Furthermore, expression of the C-terminal region of securin in pituitary tumors abrogates in vitro colony formation in soft agar and in vivo tumor formation underpinning the important proliferative role played by securin. ${ }^{34}$

In conclusion, we demonstrate abundant securin expression by immunohistochemistry and Northern blot analysis in a large series of breast tumors compared to normal breast tissues. Highest securin expression was seen in invasive breast cancer and correlated with mitotic rates, and degree of nuclear pleomorphism. This study confirms and extends our own work and that of others on the utility of securin as a marker of lymph node invasion and metastasis in solid tumors. ${ }^{12-14,35}$

This is the first study describing the immunohistochemical analysis of securin expression in a large series of tumors and confirming its use as a technique that could potentially be employed in the clinical setting. Securin represents a potential novel marker of breast cancer proliferation and analysis of breast cancer securin expression may provide important prognostic insights and portend response to treatment in breast cancer. Furthermore, given its abundance in all breast tumors examined and its key role in breast cancer cell division, securin may represent a novel subcellular therapeutic target in breast cancer.

\section{Acknowledgements}

This work was supported by the Margaret E Early Research Trust; Borden Foundation and the Doris Factor Molecular Endocrinology Laboratory. 


\section{References}

1 Greenlee R, Hill-Harmon MB, Murray $\mathrm{T}$, et al. Cancer Statistics, 2001. CA Cancer J Clin 2001;51: 15-36.

2 Allred DC, Mohsin SK, Fuqua SA. Histological and biological evolution of human premalignant breast disease. Endocr Rel Cancer 2001;8:47-61.

3 Fisher B, Costantino JP, Wickerham DL, et al. Tamoxifen for prevention of breast cancer: report of the National Surgical Adjuvant Breast and Bowel Project P-I Study. J Natl Cancer Inst 1998;90: 1371-1388.

4 Cummings SR, Eckert S, Krueger KA, et al. The effect of raloxifene on risk of breast cancer in postmenopausal women: results from the MORE randomized trial. JAMA 1999;281:2189-2197.

5 Sommer S, Fuqua SA. Estrogen receptor and breast cancer. Semin Cancer Biol 2001;11:339-352.

6 Tsongalis GJ, Ried Jr A. HER-2: the neu prognostic marker for breast cancer. Crit Rev Clin Lab Sci 2001;38:167-182.

7 Pei L, Melmed S. Isolation and characterization of a pituitary tumor-transforming gene (PTTG). Mol Endocrinol 1997;11:433-441.

8 Jallepalli PV, Waizenegger IC, Bunz F, et al. Securin is required for chromosomal stability in human cells. Cell 2001;105:445-457.

$9 \mathrm{Yu}$ R, Heaney AP, Lu W, et al. Pituitary tumor transforming gene causes aneuploidy and p53 dependent and p53 independent apoptosis. J Biol Chem 2000;275:36502-36505.

10 Zhang X, Horwitz GA, Prezant TP, et al. Structure, expression and function of hPTTG. Mol Endocrinol 1999;13:156-166

11 Ishikawa H, Heaney AP, Yu R, et al. Human pituitary tumor-transforming gene induces angiogenesis. J Clin Endocrinol Metab 2001;86:867-874.

12 Zhang X, Horwitz GA, Heaney AP, et al. Pituitary tumor transforming gene expression in pituitary adenomas. J Clin Endocrinol Metab 1999;84:761-767.

13 Heaney AP, Nelson V, Fernando M, et al. Transforming events in thyroid tumorigenesis and their association with follicular lesions. J Clin Endocrinol Metab 2001;86:5025-5032.

14 Heaney AP, Singson R, McCabe CJ, et al. Expression of pituitary tumor transforming gene in colorectal tumors. Lancet 2000;355:716-719.

15 Heaney AP, Horwitz GA, Wang Z, et al. Early involvement of estrogen-induced PTTG and FGF expression in prolactinoma pathogenesis. Nat Med 1999;5:1317-1321.

$16 \mathrm{Yu}$ R, Ren SG, Horwitz GA, et al. Pituitary tumor transforming gene regulates placental JEG-3 cell division and survival: evidence from live cell imaging. Mol Endocrinol 2000;14:1137-1146.

17 Early Breast Cancer Trialists' Collaborative Group. Ovarian ablation in early breast cancer: overview of the randomized trials. Lancet 1996;348:1189-1196.

18 Early Breast Cancer Trialists' Collaborative Group. Tamoxifen for early breast cancer: an overview of the randomized trials. Lancet 1998;351:1451-1467.
19 Early Breast Cancer Trialists' Collaborative Group. Polychemotherapy for early breast cancer: an overview of the randomized trials. Lancet 1998;352:930-942.

20 Thomas E, Hortobagyi GN. New paradigms in adjuvant systemic therapy of breast cancer. Endocr Rel Cancer 2003;10:75-89.

21 Gerdes J, Schwab U, Lemke H, et al. Cell cycle analysis of the monoclonal antibody Ki-67. J Immunol 1984; 133:1710.

22 Brown DC, Gatter KC. Monoclonal antibody Ki-67: its use in histopathology. Histopathology 1990;17: 489-503.

23 Zhou XZ, Lu PJ, Wulf G, et al. Phosphorylationdependent prolyl isomerization: a novel signaling regulatory mechanism. Cell Mol Life Sci 1999;56: 788-809.

24 Ogbagabriel S, Fernando M, Waldman F, et al. Role of human pituitary tumor transforming gene in breast cancer pathogenesis. Endocrine Society's 84th Annual Meeting, San Francisco, 2002, pp OR40-OR44.

25 Skinner KA, Silverstein MJ. The management of ductal carcinoma in situ of the breast. Endocr Rel Cancer 2001;8:33-45.

26 Toikkanen S, Pylkkanen L, Joensuu H. Invasive lobular carcinoma of the breast has better short- and long-term survival than invasive ductal carcinoma. Br J Cancer 1997;76:1234-1240.

27 Siverstein MJ, Lewinsky BS, Waisman JR, et al. Infiltrating lobular carcinoma. Is it different from infiltrating duct carcinoma? Cancer 1994;73:16731677.

28 Sastre-Garau X, Jouve M, Asselain B, et al. Infiltrating lobular carcinoma of the breast. Clinicopathologic analysis of 975 cases with reference to data on conservative therapy and metastatic patterns. Cancer 1996;77:113-120.

29 Hajitou A, Sounni NE, Devy L, et al. Down-regulation of vascular endothelial growth factor by tissue inhibitor of metalloproteinase-2: effect on in vivo mammary tumor growth and angiogenesis. Cancer Res 2001;61:3450-3457.

30 Li R, Sonik A, Stindl R, et al. Aneuploidy vs gene mutation hypothesis of cancer: recent study claims mutation but is found to support aneuploidy. Proc Natl Acad Sci USA 2000;97:3236-3241.

31 Zimonjic D, Brooks MW, Popescu N, et al. Derivation of human tumor cells in vitro without widespread genomic instability. Cancer Res 2001;61:8838-8844.

32 Nasmyth K. Segregating sister genomes: the molecular biology of chromosome separation. Science 2002;297: 559-565.

33 Pati D, Haddad BR, Haegele A, et al. Hormone-induced chromosomal instability in p53-null mammary epithelium. Cancer Res 2004;64:5608-5616.

34 Horwitz GA, Miklovsky I, Heaney AP, et al. Human pituitary tumor-transforming gene (PTTG1) motif suppresses prolactin expression. Mol Endocrinol 2003;17: 600-609.

35 Solbach C, Roller M, Fellbaum C, et al. PTTG mRNA expression in primary breast cancer: a prognostic marker for lymph node invasion and tumor recurrence. Breast 2004;13:80-81. 